\title{
Dose reduction to organs at risk with deep- inspiration breath-hold during right breast radiotherapy: a treatment planning study
}

\author{
Chloe Pandeli ${ }^{1 *}$ (D), Lloyd M. L. Smyth', Steven David² and Andrew W. See ${ }^{1}$
}

\begin{abstract}
Background: The addition of regional nodal radiation (RNI) to whole breast irradiation for high risk breast cancer improves metastases free survival and new data suggests it contributes additional benefit to overall survival. Deep inspiration breath hold (DIBH) has been shown to reduce cardiac and pulmonary dose in the context of left-sided disease treated with or without RNI, yet few studies have investigated its utility for right-breast cancer. This study investigates the potential advantages of DIBH in local and locoregional radiotherapy for right-sided breast cancer.

Methods: Free-breathing (FB) and DIBH computed tomography datasets were obtained from twenty patients who previously underwent radiotherapy for left-sided breast cancer. Ten patients were retrospectively planned for whole right breast only irradiation and ten patients were planned for irradiation to the whole breast plus ipsilateral supraclavicular (SC) nodes, with and without irradiation of the ipsilateral internal mammary nodes (IMN). Dose-volume metrics for the clinical target volume, lungs, heart, left anterior descending artery, right coronary artery (RCA) and liver were recorded. Differences between FB and DIBH plans were analysed using Wilcoxon signed-rank tests, with $P<0.05$ considered statistically significant.
\end{abstract}

Results: DIBH increased the average total lung volume compared to FB in both breast only and breast plus RNI cohorts $(P=0.001)$. For the breast only group, there was no significant improvement in any ipsilateral lung dosevolume metric between FB and DIBH. However, for the breast plus RNI group, there was an improvement in ipsilateral lung mean dose $(18.9 \pm 3.2 \mathrm{~Gy}$ to $15.9 \pm 2.3 \mathrm{~Gy}, P=0.002)$ and V20Gy $(45.3 \pm 13.3 \%$ to $32.9 \pm 9.4 \%, P=$ 0.002). In addition, DIBH significantly reduced the maximum dose to the RCA for RNI (11.6 \pm 7.2 Gy to $5.6 \pm 2.9 \mathrm{~Gy}$, $P=0.03$ ). Significant reductions in the liver V20Gy and maximum dose were observed in all cohorts during DIBH compared to FB.

Conclusions: DIBH is a promising approach for right-breast radiotherapy with considerable sparing of normal tissue, particularly when the ipsilateral IMNs are also irradiated.

Keywords: Breast cancer, Deep inspiration breath-hold, Liver, Lung, IMRT, Regional nodal irradiation, Treatment planning

\footnotetext{
* Correspondence: chloe.pandeli@icon.team

${ }^{1}$ Icon Cancer Centre, Level 4, The Epworth Centre, 32 Erin Street, Richmond,

Victoria 3121, Australia

Full list of author information is available at the end of the article
}

(c) The Author(s). 2019 Open Access This article is distributed under the terms of the Creative Commons Attribution 4.0 International License (http://creativecommons.org/licenses/by/4.0/), which permits unrestricted use, distribution, and reproduction in any medium, provided you give appropriate credit to the original author(s) and the source, provide a link to the Creative Commons license, and indicate if changes were made. The Creative Commons Public Domain Dedication waiver (http://creativecommons.org/publicdomain/zero/1.0/) applies to the data made available in this article, unless otherwise stated. 


\section{Background}

Adjuvant whole breast irradiation following lumpectomy is the standard of care for women diagnosed with early stage breast cancer. For women with node-positive or high-risk node-negative disease, the irradiation of regional nodes reduces the rate of both locoregional and distant recurrence $[1,2]$. However, the addition of regional nodal irradiation (RNI) increases the volume of underlying normal tissue exposed to radiation, increasing the risk of toxicity.

Deep inspiration breath-hold (DIBH) is a respiratory manoeuvre predominantly used to mitigate the increased risk of late cardiac toxicity for women receiving left-breast radiotherapy [3]. DIBH significantly reduces cardiac dose during whole left-breast irradiation, with or without RNI [4-8]. Lung-sparing benefits are also reported [4, 5, 9]. Importantly, the technique is highly reproducible and stable over the course of treatment $[7,10,11]$.

Despite the widespread implementation of DIBH for left-breast radiotherapy, DIBH is rarely used for rightsided treatment and there are few studies exploring the possible dosimetric advantages. Early data suggests cardiac and pulmonary benefits for right-breast cancer patients when RNI is also prescribed [12,13]. Liver-sparing is also reported [13, 14], however, data remains limited.

The aim of this treatment planning study was to quantify the dose-sparing benefits of DIBH compared to freebreathing (FB) for right-sided breast radiotherapy, with and without irradiation of the regional nodes. Dosedistributions to the lungs, heart, left anterior descending (LAD) artery, right coronary artery (RCA) and liver are reported.

\section{Methods}

\section{Patient population and study design}

Free-breathing and DIBH computed tomography (CT) scans were obtained from twenty patients originally receiving intact left-breast irradiation between January 2016 and November 2017. All patients were retrospectively planned on both DIBH and FB datasets for radiotherapy to the whole right breast $(n=10)$ or whole right breast plus ipsilateral SC nodes, with or without the ipsilateral IMNs $(n=$ 10). No extra imaging was required for the study. This retrospective study was approved by the Epworth HealthCare Human Research and Ethics Committee.

Patients were scanned in a supine position with arms raised over head and supported by a personalised vacuumfixed mould. Patients did not change position between the $\mathrm{FB}$ and DIBH scans. Patients received verbal coaching from radiation therapists, as well as visual bio-feedback, during the DIBH scan. The Real-time Position Management ${ }^{\mathrm{Tu}}$ (RPM) system (Varian Medical Systems, Palo Alto, CA) was used to monitor breathing during both FB and DIBH scans via a reflective marker box placed at the level of the xiphoid process. All patients were able to hold their breath for greater than twenty seconds to accommodate the scan during DIBH. The CT scan length was from the level of the C3 vertebra to $5 \mathrm{~cm}$ inferior to the infra-mammary fold with a CT slice thickness of $3 \mathrm{~mm}$. Scans were imported into the Eclipse ${ }^{\mathrm{mi}}$ (Varian) planning system for volume delineation and treatment planning.

\section{Target volume and organ at risk delineation}

Target volumes were delineated by one radiation oncologist and independently peer reviewed by a second radiation oncologist prior to treatment planning. The nodal clinical target volumes (CTVs) were defined according to ESTRO consensus guidelines [15]. The breast CTV was defined as the visible breast tissue on CT cropped $5 \mathrm{~mm}$ from the skin surface. The planning target volume (PTV) was a $5 \mathrm{~mm}$ isotropic expansion of the CTV which was subsequently cropped $5 \mathrm{~mm}$ from the skin surface.

Contours for both lungs were generated using an automated segmentation tool and adjusted manually where necessary. The liver and contralateral breast were contoured manually. The heart, LAD and RCA were contoured manually based on the University of Michigan Cardiac Atlas [16]. As patients were scanned without contrast, a $4 \mathrm{~mm}$ margin was used for the LAD and RCA contours.

\section{Treatment planning}

FB and DIBH treatment plans were generated for each patient by one radiation therapist to ensure plan quality and consistency across all patients. The prescription dose for all plans was $40 \mathrm{~Gy}$ in 15 fractions. Target coverage criteria were in accordance with ICRU recommendations as follows; maximum dose not exceeding $107 \%$ of the prescribed dose, coverage of the PTV by the 95\% isodose, mean dose to the PTV between 100 and $102 \%$ of the prescription dose.

Breast only plans were planned with a three-dimensional conformal technique using a tangential beam arrangement, predominantly using a $6 \mathrm{MV}$ photon beam energy. Lowweighted $10 \mathrm{MV}$ sub-fields were used where necessary to achieve adequate target coverage. Whole breast plus ipsilateral SC (level III and level IV nodes) plans were generated similarly to the breast only plans, however, with the inclusion of an anterior-oblique field for the SC nodal region. The breast plus ipsilateral SC and IMN group (subsequently referred to as breast plus RNI) were inverse planned using six intensity modulated radiation therapy (IMRT) fields with a beam energy of $6 \mathrm{MV}$. Dose calculation was performed in Eclipse $^{\text {Tx }}$ (Varian Medical Systems) with the Anisotropic Analytical Algorithm (Version 13.6.26).

The dose-volume objectives for the lungs and heart were aligned to QUANTEC guidelines as follows [17]; lung V20Gy < 30\% and mean dose <20Gy, heart V30Gy < 
$46 \%$, and mean dose $<26$ Gy. The maximum dose to the heart was to be kept as low as possible. The mean dose to the contralateral left breast was restricted to less than 2 Gy.

\section{Plan evaluation and statistical analysis}

Dose-volume histograms (DVHs) were generated for all target volumes and organs at risk (OARs) on the FB and DIBH plans. The following dose-volume metrics were recorded: CTV; mean dose and volume receiving $95 \%$ of the prescription dose (V95\%), total and ipsilateral lung; mean dose, volume receiving 5 Gy (V5Gy) and 20 Gy (V20Gy) and total lung volume, heart; mean dose and maximum dose, LAD; mean dose and maximum dose, RCA; mean dose and maximum dose, and liver; maximum dose and V20Gy.

All analyses were performed using the XLSTAT software package (version 2019.1.1; XLSTAT, New York, NY). Data are presented as mean values \pm standard deviation
(SD). Wilcoxon signed-rank tests were used to analyse differences in the dose-volume constraints achieved between the FB and DIBH plans. $P<0.05$, two-tailed, was considered statistically significant.

\section{Results}

Dose-volume metrics for target volumes and OARs are summarised in Table 1. Three sets of data were collected; DIBH versus FB for whole right breast only treatment $(n=10)$, right breast plus SC only $(n=10)$ and right breast plus RNI $(n=10)$. A representative DVH comparing $\mathrm{FB}$ and $\mathrm{DIBH}$ plans for a patient receiving breast only irradiation is shown in Fig. 1a. The DVHs for breast plus SC and breast plus RNI plans, from the same patient, are shown in Fig. 1b and c, respectively. There was no difference in plan quality between DIBH and FB plans for all groups in terms of target coverage (Table 1). All plans, under both breathing conditions, met the target coverage criteria.

Table 1 Dose-volume metrics for DIBH and FB treatment plans

\begin{tabular}{|c|c|c|c|c|c|c|c|c|c|}
\hline \multirow[t]{2}{*}{ Metric } & \multicolumn{3}{|l|}{ Breast Only } & \multicolumn{3}{|l|}{ Breast + SC only } & \multicolumn{3}{|l|}{ Breast + RNI } \\
\hline & $\overline{F B}$ & $\mathrm{DIBH}$ & $P$ value & $\overline{F B}$ & $\mathrm{DIBH}$ & $P$ value & $\overline{F B}$ & $\mathrm{DIBH}$ & $P$ value \\
\hline \multicolumn{10}{|l|}{ CTV } \\
\hline Mean (Gy) & $40.9 \pm 0.4$ & $40.9 \pm 0.4$ & 0.6 & $40.9 \pm 0.3$ & $40.8 \pm 0.3$ & 0.8 & $40.6 \pm 0.2$ & $40.6 \pm 0.2$ & 0.5 \\
\hline V95\% (\%) & $99.4 \pm 0.7$ & $99.5 \pm 0.8$ & 1 & $99.4 \pm 0.4$ & $98.7 \pm 1.5$ & 0.3 & $100.0 \pm 0.03$ & $99.9 \pm 0.2$ & 1 \\
\hline \multicolumn{10}{|l|}{ Lung (total) } \\
\hline Mean (Gy) & $2.4 \pm 0.5$ & $2.3 \pm 0.8$ & 0.3 & $3.5 \pm 0.5$ & $3.1 \pm 0.5$ & 0.01 & $10.8 \pm 1.7$ & $8.9 \pm 1.2$ & 0.002 \\
\hline V20Gy (\%) & $4.1 \pm 0.5$ & $4.0 \pm 1.9$ & 0.4 & $6.7 \pm 1.2$ & $5.4 \pm 1.2$ & 0.02 & $25.2 \pm 7.6$ & $17.9 \pm 5.1$ & 0.002 \\
\hline V5Gy (\%) & $9.6 \pm 2.3$ & $8.7 \pm 3.2$ & 0.3 & $13.9 \pm 2.3$ & $12.4 \pm 1.6$ & 0.02 & $51.0 \pm 3.5$ & $47.4 \pm 3.2$ & 0.004 \\
\hline Volume $\left(\mathrm{cm}^{3}\right)$ & $2613.2 \pm 728.1$ & $4467.6 \pm 649.0$ & 0.001 & $2714.8 \pm 595.0$ & $4452.0 \pm 591.5$ & 0.001 & $2714.8 \pm 595.0$ & $4452.0 \pm 591.5$ & 0.001 \\
\hline \multicolumn{10}{|l|}{ Lung (ipsilateral) } \\
\hline Mean (Gy) & $4.4 \pm 1.0$ & $4.3 \pm 1.5$ & 0.4 & $6.3 \pm 0.9$ & $5.6 \pm 0.9$ & 0.05 & $18.9 \pm 3.2$ & $15.9 \pm 2.3$ & 0.002 \\
\hline V20Gy (\%) & $7.6 \pm 2.3$ & $7.5 \pm 3.6$ & 0.4 & $12.0 \pm 2.2$ & $9.8 \pm 2.2$ & 0.03 & $45.3 \pm 13.3$ & $32.9 \pm 9.4$ & 0.002 \\
\hline V5Gy (\%) & $17.6 \pm 4.3$ & $16.4 \pm 6.0$ & 0.3 & $25.1 \pm 4.0$ & $22.7 \pm 3.1$ & 0.06 & $91.6 \pm 5.6$ & $86.7 \pm 7.1$ & 0.01 \\
\hline \multicolumn{10}{|l|}{ Liver } \\
\hline Max. (Gy) & $22.3 \pm 15.7$ & $9.2 \pm 13.6$ & 0.006 & $32.4 \pm 11.0$ & $10.4 \pm 10.8$ & 0.001 & $31.3 \pm 13.3$ & $15.3 \pm 9.8$ & 0.002 \\
\hline V20Gy (cc) & $9.2 \pm 18.9$ & $1.8 \pm 5.6$ & 0.03 & $10.8 \pm 17.2$ & $0.8 \pm 2.6$ & 0.001 & $24.3 \pm 38.4$ & $0.8 \pm 2.2$ & 0.02 \\
\hline \multicolumn{10}{|l|}{ Heart } \\
\hline Mean (Gy) & $0.2 \pm 0.1$ & $0.2 \pm 0.1$ & 0.7 & $0.3 \pm 0.1$ & $0.4 \pm 0.1$ & 0.2 & $2.6 \pm 0.8$ & $2.1 \pm 0.7$ & 0.09 \\
\hline Max. (Gy) & $2.4 \pm 0.5$ & $2.5 \pm 0.4$ & 0.5 & $1.6 \pm 0.5$ & $1.7 \pm 0.4$ & 0.9 & $27.8 \pm 7.6$ & $21.5 \pm 6.6$ & 0.02 \\
\hline \multicolumn{10}{|l|}{$\angle A D$} \\
\hline Mean (Gy) & $0.03 \pm 0.02$ & $0.03 \pm 0.02$ & 0.7 & $0.1 \pm 0.04$ & $0.1 \pm 0.03$ & 0.2 & $0.5 \pm 0.2$ & $0.6 \pm 0.2$ & 0.1 \\
\hline Max (Gy) & $0.1 \pm 0.1$ & $0.1 \pm 0.1$ & 0.4 & $0.2 \pm 0.1$ & $0.2 \pm 0.1$ & 0.1 & $1.2 \pm 0.8$ & $1.2 \pm 0.7$ & 0.9 \\
\hline \multicolumn{10}{|l|}{$R C A$} \\
\hline Mean (Gy) & $0.7 \pm 0.3$ & $0.6 \pm 0.3$ & 0.4 & $0.9 \pm 0.2$ & $1.0 \pm 0.4$ & 0.5 & $5.0 \pm 2.5$ & $3.5 \pm 1.9$ & 0.1 \\
\hline $\operatorname{Max}(G y)$ & $1.1 \pm 0.4$ & $1.0 \pm 0.5$ & 0.5 & $1.6 \pm 0.5$ & $1.7 \pm 0.4$ & 0.7 & $11.6 \pm 7.2$ & $5.6 \pm 2.9$ & 0.03 \\
\hline
\end{tabular}

All data $(n=10)$ are presented as mean \pm SD

Abbreviations: CTV clinical target volume, DIBH deep inspiration breath-hold, FB free-breathe, $L A D$ left anterior descending artery, $R C A$ right coronary artery, $R N I$ regional nodal irradiation, SC supra-clavicular 

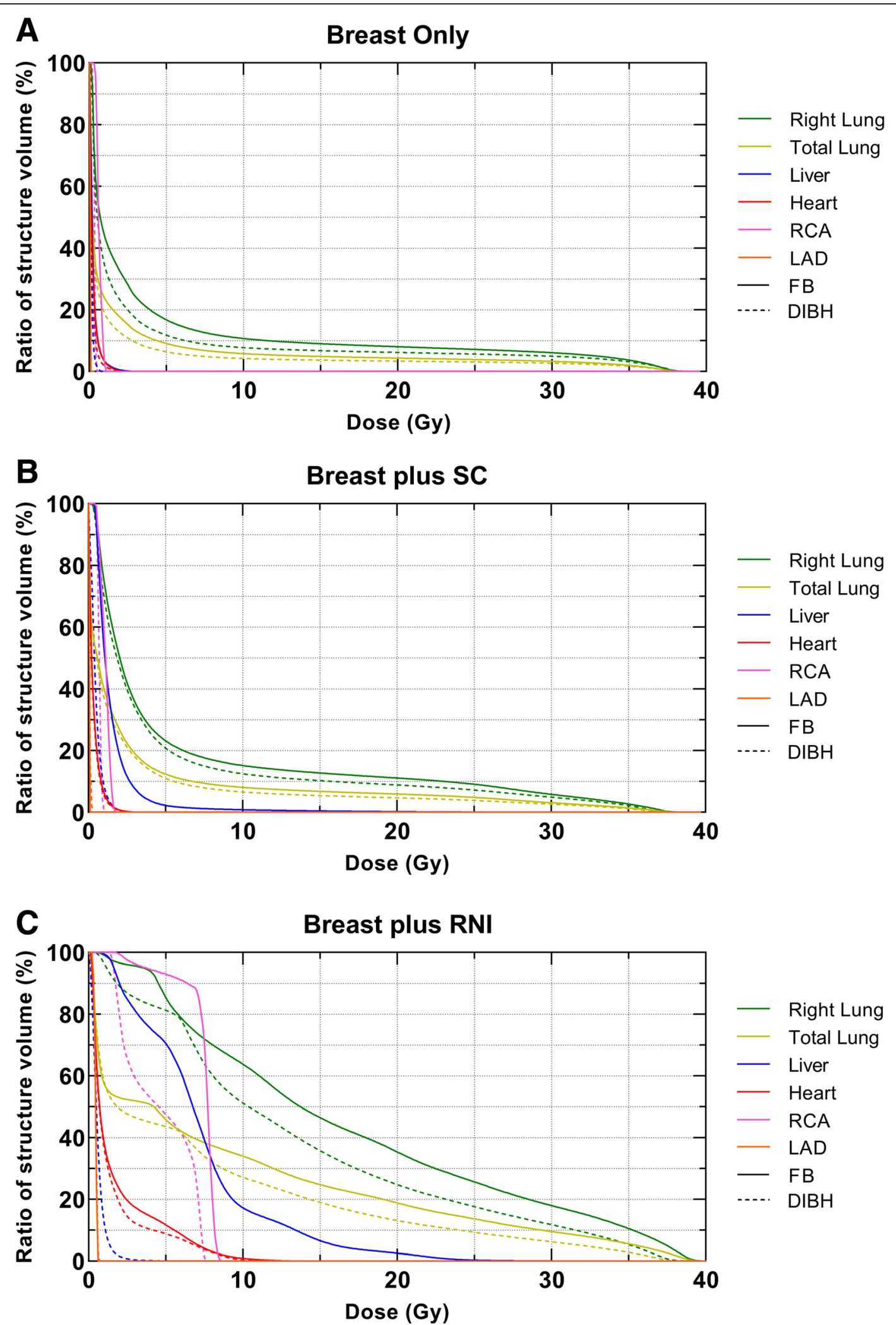

Fig. 1 Representative DVHs for OARs when comparing FB and DIBH for breast only (a), breast plus SC (b) and breast plus RNI (c). DVHs taken from one representative patient per group 


\section{Pulmonary dose}

For all three cohorts, there was a similar increase in total lung volume for DIBH compared to FB. The total lung volume for the breast only group increased from $2613.2 \pm 728.1 \mathrm{~cm}^{3}$ (FB) to $4467.6 \pm 649.0 \mathrm{~cm}^{3}$ (DIBH) ( $P=0.001)$ while for the breast plus RNI and breast plus SC groups, the increase was from $2714.8 \pm 595.0 \mathrm{~cm}^{3}$ to $4452 \pm 591.5 \mathrm{~cm}^{3}(P=0.001)$. There was no significant difference in any total or ipsilateral lung dose-volume metric between FB and DIBH in the breast-only group. By contrast, DIBH was associated with significant pulmonary sparing for the breast plus RNI plans. All ten patients in the breast plus RNI group recorded decreases in the mean dose and V20Gy for both total and ipsilateral lung volumes. The ipsilateral lung V20Gy decreased from $45.3 \pm 13.3 \%$ to $32.9 \pm 9.4 \%$ for DIBH plans $(P=$ 0.002) (Fig. 2), with the largest reduction being from 67.2 to $42.7 \%$. The ipsilateral lung V5Gy decreased from $91.6 \pm 5.6 \%$ to $86.7 \pm 7.1 \%$ with DIBH $(P=0.01)$. A significant reduction in the ipsilateral lung V20Gy was also achieved in the breast plus SC group, with 8 out of the 10 patients recording a decrease in this metric.

Two patients in the breast only group had an increase in ipsilateral lung V20Gy with DIBH, however, the constraint of V20Gy $<30 \%$ was still achieved. Nine out of ten patients in the breast plus RNI group had an initial ipsilateral lung V20Gy > 30\% during FB, with four out of ten meeting this constraint when utilising DIBH.

\section{Liver dose}

Statistically significant reductions in the liver V20Gy and maximum dose were observed in both breast-only and breast plus RNI plans during DIBH compared to FB
(Fig. 3). The greatest decrease in liver V20Gy was in the breast plus RNI group, with a reduction from $24.3 \pm 38.4$ cc to $0.8 \pm 2.2 \mathrm{cc}(P=0.02)$. The V20Gy was reduced to zero for four out of six patients in the right breast only group and four out of eight patients in the breast plus RNI group. Movement of the liver inferiorly during DIBH compared to FB is shown in Fig. 4 for a pair of breast plus RNI plans from the same patient.

\section{Cardiac dose}

As expected, heart dose was very low for most patients in breast only, breast plus SC and RNI groups. The mean and maximum heart doses for patients in the breast-only group were comparable between FB and DIBH plans. However, a significant decrease in the maximum heart dose was seen for the breast plus RNI plans $(27.8 \pm 7.6$ Gy versus $21.5 \pm 6.6 \mathrm{~Gy}, P=0.02)$.

In addition, a significant decrease in the maximum RCA dose was observed for the breast plus RNI group, where DIBH decreased the average maximum dose from $11.6 \pm 7.2$ Gy to $5.6 \pm 2.9 \mathrm{~Gy}(P=0.03)$. The largest recorded reduction in maximum RCA dose for the RNI group was $21 \mathrm{~Gy}(\mathrm{FB}=26 \mathrm{~Gy}, \mathrm{DIBH}=5 \mathrm{~Gy})$. RCA dose was low for the breast only and breast plus $\mathrm{SC}$ group in both FB and DIBH.

The dose to the LAD artery was universally low across all groups, with no significant differences between DIBH and FB.

\section{Discussion}

This study supports the use of DIBH for right-sided breast cancer patients, particularly those undergoing concomitant RNI. For patients receiving RNI, there was

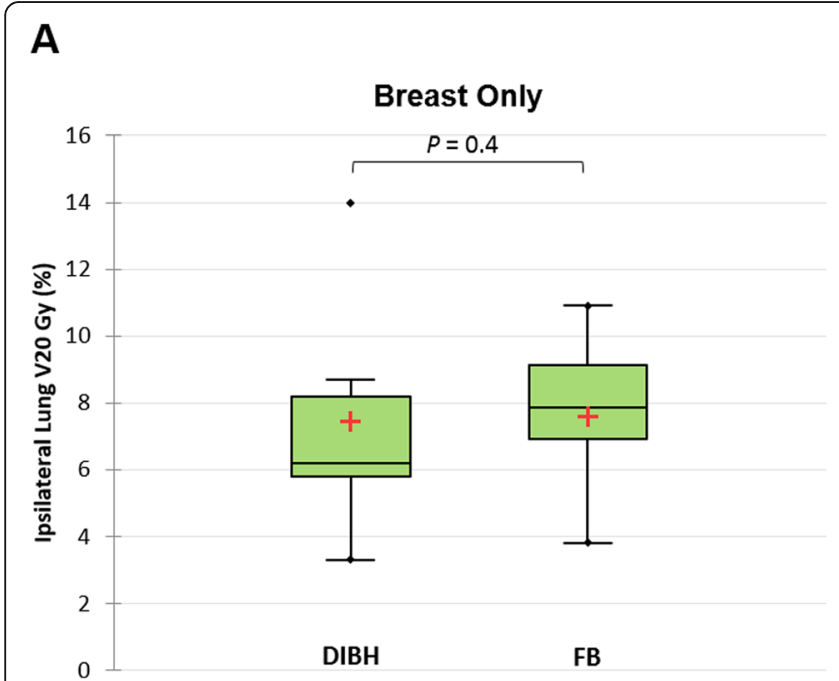

B

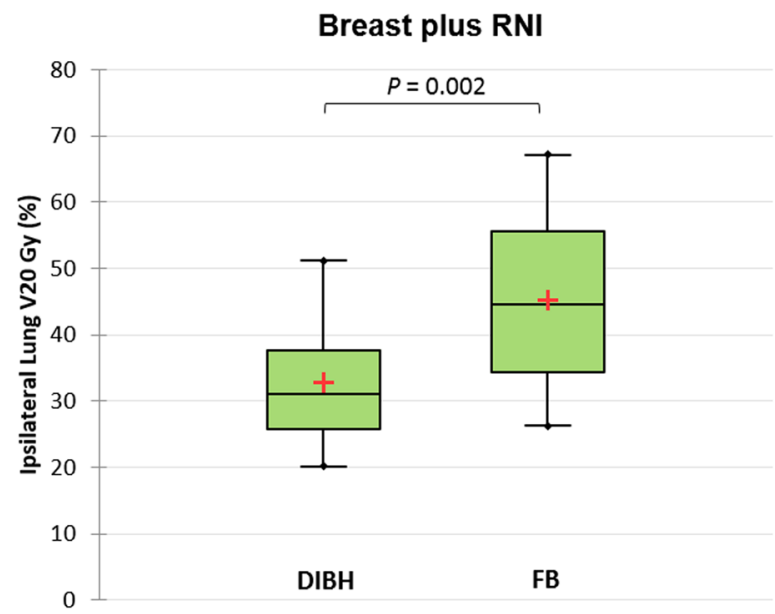

Fig. 2 Box-plots of ipsilateral lung V20Gy for DIBH versus FB in right breast only (a) and right breast plus RNI (b) groups. A significant difference in V20Gy was seen for the breast plus RNI group only. The red cross indicates the group mean and the whiskers extend to 1.5 times the IQR, with $n=10$ per group 

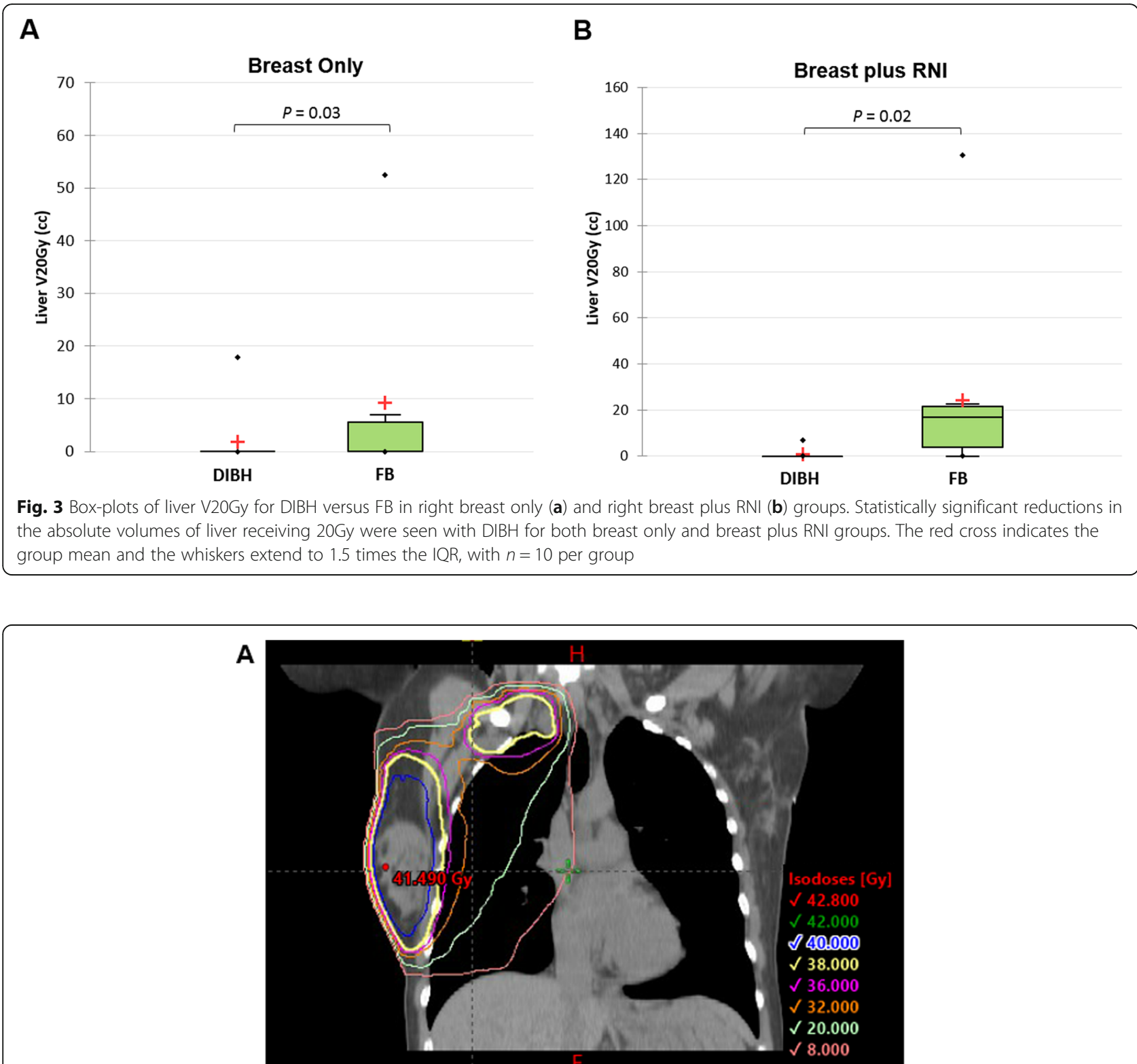

B

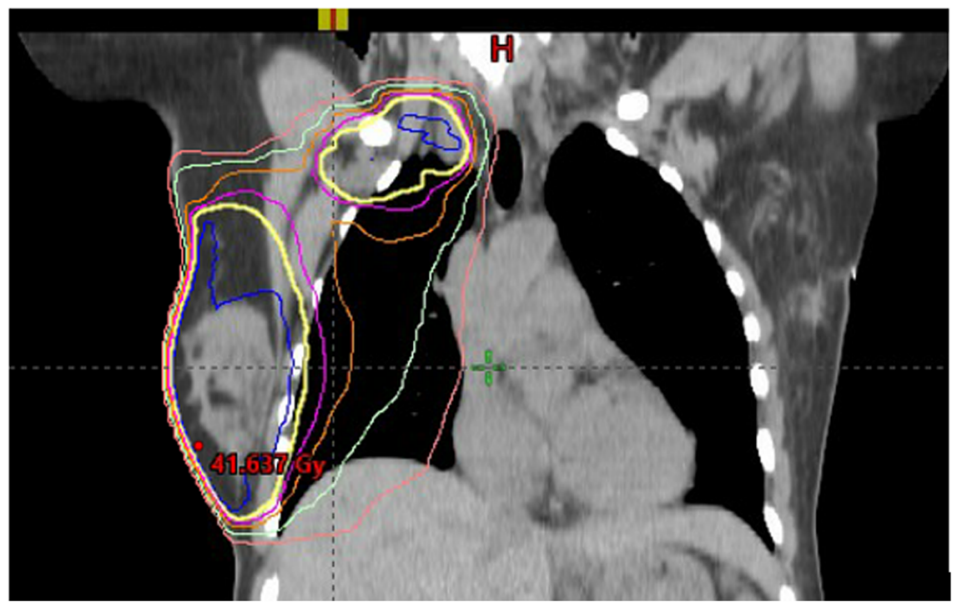

Fig. 4 Coronal CT slices demonstrating the superior-inferior liver displacement between DIBH (a) and FB (b) for a breast plus RNI patient 
a $12 \%$ and nearly $5 \%$ absolute reduction in the volume of ipsilateral lung receiving $20 \mathrm{~Gy}$ and $5 \mathrm{~Gy}$, respectively. A significant reduction in both the maximum liver dose and the volume of liver receiving $20 \mathrm{~Gy}$ was also observed for right-breast only, breast plus SC and breast plus RNI patients. The maximum dose to the RCA was also significantly reduced for the RNI group.

The rate of radiation-induced pneumonitis (RP) following breast radiotherapy in the modern era is low (1 to $5 \%$ ) with reductions in lung-function being more common [18, 19]. Nevertheless, increasing pulmonary dose is associated with a higher rate and severity of RP [20]. Unsurprisingly, the rate of RP is higher for breast cancer patients receiving RNI compared to those receiving whole breast radiotherapy only [1, 21, 22]. These observations further support the prudence of utilising DIBH during right-sided breast radiotherapy to reduce pulmonary exposure, which is particularly relevant when the regional nodes are also irradiated. DIBH not only reduces the percentage volume of lung receiving $20 \mathrm{~Gy}$ and 5 Gy, but also reduces lung tissue density [23] which could further contribute to a lower normal tissue complication probability [24].

The absolute reduction in ipsilateral lung V20Gy with DIBH in our study (12.4\%) was greater than previously reported for right-sided breast cancer patients receiving additional RNI. In this subset of patients, Essers et al. [12] and Conway et al. [13] showed an average absolute reduction in ipsilateral lung V20Gy of $7.5 \%$ and of $7.8 \%$, respectively. Our report of a greater pulmonary benefit could be explained by differences in the FB ipsilateral lung V20Gy related to the chosen planning technique. Essers et al. [12] adopted a volumetric modulated arc therapy approach using partial arcs [25] when including the internal mammary and peri-clavicular nodes while Conway et al. [13] used a conformal approach involving wide tangential fields. During left breast irradiation with RNI, the reduction in ipsilateral lung V20Gy is reported to be up to $11.8 \%$ [5]. Our finding of a $12.4 \%$ reduction in ipsilateral lung V20Gy, with additional RNI, is therefore not unreasonable.

In addition to reducing high-dose pulmonary exposure during RNI, our results show that DIBH also leads to a small yet significant reduction in the proportion of ipsilateral lung receiving $5 \mathrm{~Gy}$. For the complex geometry of the CTV in breast radiotherapy with RNI, intensity modulation optimises dose conformity, homogeneity and target coverage. In our study, conformal coverage of the CTV by the $95 \%$ of the prescription dose was achieved. However, the superior geometric target coverage associated with IMRT compared to 3D conformal techniques is offset by increased low-dose exposure due to additional beam angles, monitor units, and inter-leaf radiation leakage. In a meta-analysis of 762,468 breast cancer patients, Grantzau and Overgaard [26] found a significantly increased risk of secondary cancer following radiotherapy for breast cancer. The excess risk of secondary lung cancer in patients receiving radiotherapy compared to those who did not was 39 and $66 \%$ at five years and fifteen years following treatment, respectively [26]. Furthermore, the increased use of modern intensity modulated approaches is estimated to nearly double the incidence of secondary malignancies from 1 to $1.75 \%$ at ten years following treatment [27]. Therefore, RNI delivered by IMRT - which is the standard at our institution - warrants the utilisation of DIBH to reduce the volume of lung exposed to low-dose irradiation and to potentially offset the additional risk of secondary malignancy.

One of the potentially important findings of our study is the significant reduction in hepatic dose with DIBH for both right-breast only and breast plus RNI groups. Radiation-induced liver damage (RILD) is not commonly associated with breast radiotherapy, but rather with treatments involving the abdomen, lower lobe of the right lung, or distal oesophagus. The dose delivered to the liver during right breast radiotherapy is substantially lower than the mean dose (30-32 Gy) considered to be a significant predictor for a $5 \%$ chance of RILD [28]. While the clinical significance of hepatic dose during right-breast radiotherapy remains to be established, it is in the best interests of patients, and in alignment with the 'as low as reasonably achievable' (ALARA) principle, to reduce dose to all potential organs at risk as much as possible. Our data, alongside previous studies [13, 14, 29], clearly demonstrates a role for DIBH in reducing hepatic dose which is achieved through increasing the superiorinferior separation between the CTV and the liver as the lungs expand (Fig. 4).

Although cardiac-sparing is most relevant to leftbreast radiotherapy, the irradiation of the ipsilateral IMN in right-sided breast radiotherapy can increase the exposure of the heart to radiation. In our study, DIBH led to a statistically significant reduction in maximum heart dose of 6 Gy during breast plus RNI $(P=0.02)$. A modest but non-significant reduction in mean heart dose of $0.5 \mathrm{~Gy}(P=0.09)$ was observed, with reductions up to 2 Gy recorded, which could be attributable to unfavourable cardiac anatomy. Darby et al. [3] previously demonstrated that the risk of major coronary events increases linearly with mean heart dose at a rate of $7.4 \%$ per Gray, with no threshold dose. Therefore, a subset of women receiving right breast radiotherapy and RNI - particularly those with unfavourable cardiac anatomy or a background of cardiac comorbidities - could benefit from DIBH.

In addition, our study observed a significant reduction in the maximum dose to the RCA with DIBH in the breast plus RNI group. While there is limited data on 
the effects of radiation on the RCA, a recent study by Altinok et al. [30] suggests that high doses to the proximal RCA could predispose patients to coronary artery disease. Therefore, our study provides further support for implementing DIBH for right breast radiotherapy where RNI is prescribed.

The case for utilising DIBH in right-sided breast plus regional node radiotherapy is supported by its ease of use, reproducibility and cost-effectiveness. Commercial [10, 31], non-commercial [32] and combination [33, 34] DIBH solutions yield systematic and random set-up errors of approximately $2 \mathrm{~mm}$ or less $[10,11,35]$. The addition of DIBH to breast radiotherapy can be expected to extend treatment appointments by three to five minutes, with minimal changes to overall patient throughout reported [33]. Nevertheless, the utility of DIBH should be considered against the imaging dose of an extra planning CT scan (one each in FB and DIBH) and the patient's ability to comply with breath-hold requirements. There are also cases where DIBH may not lead to a material improvement in dose-distribution. In our study, three of twenty patients had an increase in ipsilateral lung V20Gy with DIBH. As such, there is a need to prospectively identify patients who will dosimetrically benefit from DIBH. Anatomic factors such as tumour bed location, lung volume, and the distance of the heart from the chest wall can optimise the selection of patients for DIBH during left-breast radiotherapy, without the need for a CT scan during DIBH [36, 37]. Future studies are required to identify and validate appropriate selection criteria for the use of DIBH during right-sided breast radiotherapy.

There are limitations to our study that should be noted. Firstly, the value of isolated dose-volume metrics as predictors of pulmonary toxicity is contentious given the relative lack of data specific to breast irradiation, the relatively low rate of $\mathrm{RP}$, and the absence of a clear threshold dose for RP [19]. Ho et al. [38] recently found that the lung V20Gy and V5Gy were not predictive for grade three RP for breast cancer patients receiving addition RNI. In contrast, Lind et al. [39] demonstrated through multivariate modelling that the ipsilateral lung V20Gy predicted for radiologic and symptomatic pneumonitis, as well as pulmonary function. In our study, we chose the ipsilateral and total lung V20Gy and mean dose, in alignment with previous studies and QUANTEC recommendations [19]. The V5Gy was analysed to assess the extent of the low dose region, which is particularly relevant for IMRT and the risk of secondary malignancy [26].

Secondly, the results presented in our study are specific to the planning techniques and dose prescriptions used as standard by our department. The magnitude of the dosimetric benefits of DIBH for right-sided breast cancer will vary between intensity-modulated, static, and arc-based approaches, and vary according to institutional target contouring practices and dose-volume objectives. We used the ESTRO contouring guidelines [15] for defining the breast and regional node target volumes. Resultantly, and similarly to Conway et al. [13], the ipsilateral lung V20Gy for both FB and DIBH plans (breast plus RNI) were relatively high, exceeding 30\% for six out of ten patients and $40 \%$ for two patients, even after DIBH. In our department, a compromise would be made to reduce the ipsilateral lung V20Gy by either reducing chest wall coverage or accepting $80 \%$ isodose coverage of the internal mammary node CTV. The benefit of DIBH in right-sided treatment might be diminished in institutions where the FB ipsilateral lung V20Gy is initially lower, which could be the case if less conservative contouring guidelines are followed or clinical compromises on target coverage are made.

Thirdly, only one radiation therapist generated the treatment plans for all patients. While this has the advantage of eliminating the influence of inter-planner variation between treatment plans, there may still be the possibility for planning bias between different patients. Future planning studies could utilise knowledge-based planning solutions such as RapidPlan ${ }^{\text {Tx }}$ (Varian Medical Systems) to reduce the variability in plans introduced by inter- and intra-planner biases [40].

Finally, this was a retrospective planning study with a relatively small number of patients in each group. Future prospective studies, with larger cohorts, should be designed to more robustly determine the dosimetric benefits of adding DIBH to right-breast radiotherapy, and ultimately, to identify optimal candidates for the technique. Long-term follow-up of toxicity and clinical outcomes will be essential in establishing the true value of DIBH in the context of right-sided breast radiotherapy.

\section{Conclusion}

We have shown that DIBH could lead to substantial sparing of normal-tissue during radiotherapy for rightsided breast cancer patients, particularly those prescribed RNI to reduce the risk of disease recurrence. For patients receiving treatment to the whole right breast plus RNI, the ipsilateral lung V20Gy, V5Gy and mean dose were significantly reduced, along with a significant reduction in the maximum dose to the RCA. Significant reductions in the liver V20Gy and maximum dose were observed for right sided breast cancer patients regardless of whether the ipsilateral IMNs were targeted. Future prospective studies are required to identify which patients will benefit most from DIBH during right-breast radiotherapy and whether improvements to dose-distribution will translate into improved toxicity outcomes. 


\section{Abbreviations}

CT: Computed tomography; CTV: Clinical target volume; DIBH: Deep inspiration breath hold; FB: Free-breathing; IMN: Internal mammary nodes; IMRT: Intensity modulated radiotherapy; LAD: Left anterior descending artery; OAR: Organ at risk; PTV: Planning target volume; RCA: Right coronary artery; RILD: Radiation-induced liver damage; RNI: Regional nodal irradiation; RP: Radiation-induced pneumonitis; SC: Supraclavicular; SD: Standard deviation; V20Gy: Volume receiving at least 20 Gy; V25Gy: Volume receiving at least 25 Gy; V5Gy: Volume receiving at least 5 Gy; V95\%: Volume receiving $95 \%$ of the prescription dose

\section{Acknowledgements}

The authors would like to thank Skye Nolan for assistance with data analysis and David Jolly for providing constructive comments on the manuscript.

\section{Authors' contributions}

CP and AWS conceived the idea for this study. AWS and SD delineated the target volumes and CP performed the treatment planning. CP and LS analysed the data and drafted the manuscript. All authors read and approved the final manuscript.

\section{Funding}

There is no supporting funding for the research or any one of the authors.

\section{Availability of data and materials}

The datasets generated and analysed during the current study are available from the corresponding author on reasonable request.

\section{Ethics approval and consent to participate}

This retrospective study was approved by the Epworth HealthCare Human Research and Ethics Committee, with patient consent not required (LR 23315).

\section{Consent for publication}

Not applicable.

\section{Competing interests}

The authors declare that they have no competing interests.

\section{Author details}

${ }^{1}$ Icon Cancer Centre, Level 4, The Epworth Centre, 32 Erin Street, Richmond, Victoria 3121, Australia. ${ }^{2}$ Icon Cancer Centre, Mulgrave, Victoria 3170, Australia.

Received: 3 June 2019 Accepted: 26 November 2019 Published online: 10 December 2019

\section{References}

1. Whelan TJ, Olivotto IA, Parulekar WR, Ackerman I, Chua BH, Nabid A, et al. Regional nodal irradiation in early-stage breast cancer. N Engl J Med. 2015; 373:307-16.

2. Poortmans PM, Collette S, Kirkove C, Van Limbergen E, Budach V Struikmans $\mathrm{H}$, et al. Internal mammary and medial supraclavicular irradiation in breast cancer. N Engl J Med. 2015;373:317-27.

3. Darby SC, Ewertz M, McGale P, Bennet AM, Blom-Goldman U, Brønnum D, et al. Risk of ischemic heart disease in women after radiotherapy for breast cancer. N Engl J Med. 2013;368:987-98.

4. Swanson T, Grills IS, Ye H, Entwistle A, Teahan M, Letts N, et al. Six-year experience routinely using moderate deep inspiration breath-hold for the reduction of cardiac dose in left-sided breast irradiation for patients with earlystage or locally advanced breast cancer. Am J Clin Oncol. 2013;36:24-30.

5. Hjelstuen MHB, Mjaaland I, Vikström J, Dybvik Kl. Radiation during deep inspiration allows loco-regional treatment of left breast and axillary-, supraclavicular- and internal mammary lymph nodes without compromising target coverage or dose restrictions to organs at risk. Acta Oncol. 2012;51: 333-44

6. Hayden AJ, Rains M, Tiver K. Deep inspiration breath hold technique reduces heart dose from radiotherapy for left-sided breast cancer. J Med Imaging Radiat Oncol. 2012;56(4):464-72.

7. Schönecker S, Walter F, Freislederer P, Marisch C, Scheithauer H, Harbeck N, et al. Treatment planning and evaluation of gated radiotherapy in left-sided breast cancer patients using the Catalyst ${ }^{\mathrm{TM}} / \mathrm{Sen}$ tinel ${ }^{\mathrm{TM}}$ system for deepinspiration breath-hold (DIBH). Radiat Oncol. 2016;11:143.

8. Simonetto C, Eidemüller M, Gaasch A, Pazos M, Schönecker S, Reitz D, et al. Does deep inspiration breath-hold prolong life? Individual risk estimates of ischemic heart disease after breast cancer radiotherapy. Radiother Oncol. 2019;131:202-7.

9. Dumane VA, Saksornchai K, Zhou Y, Hong L, Powell S, Ho AY. Reduction in low-dose to normal tissue with the addition of deep inspiration breath hold (DIBH) to volumetric modulated arc therapy (VMAT) in breast cancer patients with implant reconstruction receiving regional nodal irradiation. Radiat Oncol. 2018;13:187.

10. McIntosh A, Shoushtari AN, Benedict SH, Read PW, Wijesooriya K. Quantifying the reproducibility of heart position during treatment and corresponding delivered heart dose in voluntary deep inhalation breath hold for left breast cancer patients treated with external beam radiotherapy. Int J Radiat Oncol Biol Phys. 2011;81:569-76.

11. Betgen A, Alderliesten T, Sonke J-J, van Vliet-Vroegindeweij C, Bartelink H, Remeijer P. Assessment of set-up variability during deep inspiration breath hold radiotherapy for breast cancer patients by 3D-surface imaging. Radiother Oncol. 2013;106:225-30.

12. Essers M, Poortmans PM, Verschueren $\mathrm{K}$, Hol S, Cobben DCP. Should breathing adapted radiotherapy also be applied for right-sided breast irradiation? Acta Oncol. 2016;55:460-5.

13. Conway JL, Conroy L, Harper L, Scheifele M, Li H, Smith WL, et al. Deep inspiration breath-hold produces a clinically meaningful reduction in ipsilateral lung dose during locoregional radiation therapy for some women with right-sided breast cancer. Pract Radiat Oncol. 2017;7:147-53.

14. Rice L, Harris S, Green MML, Price PM. Deep inspiration breath-hold (DIBH) technique applied in right breast radiotherapy to minimize liver radiation. BJR Case Rep. 2015;1:20150038.

15. Offersen BV, Boersma LJ, Kirkove C, Hol S, Aznar MC, Biete Sola A, et al. ESTRO consensus guideline on target volume delineation for elective radiation therapy of early stage breast cancer. Radiother Oncol. 2015;114:3-10.

16. Feng M, Moran JM, Koelling T, Chughtai A, Chan JL, Freedman L, et al. Development and validation of a heart atlas to study cardiac exposure to radiation following treatment for breast cancer. Int J Radiat Oncol Biol Phys. 2011;79:10-8.

17. Marks LB, Yorke ED, Jackson A, Ten Haken RK, Constine LS, Eisbruch A, et al. Use of normal tissue complication probability models in the clinic. Int J Radiat Oncol Biol Phys. 2010;76(Suppl 3):10-9.

18. Marks LB, Yu X, Vujaskovic Z, Small W, Folz R, Anscher MS. Radiationinduced lung injury. Semin Radiat Oncol. 2003;13:333-45.

19. Marks LB, Bentzen SM, Deasy JO, Kong F-M, Bradley JD, Vogelius IS, et al. Radiation dose-volume effects in the lung. Int J Radiat Oncol Biol Phys. 2010;76(Suppl 3):70-6.

20. Rothwell RI, Kelly SA, Joslin CAF. Radiation pneumonitis in patients treated for breast cancer. Radiother Oncol. 1985:4:9-14.

21. Matzinger O, Heimsoth I, Poortmans P, Collette L, Struikmans H, Bogaert $W V D$, et al. Toxicity at three years with and without irradiation of the internal mammary and medial supraclavicular lymph node chain in stage I to III breast cancer (EORTC trial 22922/10925). Acta Oncol. 2010;49:24-34.

22. Choi J, Kim YB, Shin KH, Ahn S-J, Lee H-S, Park W, et al. Radiation pneumonitis in association with internal mammary node irradiation in breast cancer patients: an ancillary result from the KROG 08-06 study. J Breast Cancer. 2016;19:275-82.

23. Hedin E, Bäck A, Chakarova R. Impact of lung density on the lung dose estimation for radiotherapy of breast cancer. Phys Imaging Radiat Oncol. 2017;3:5-10

24. Rosenzweig KE, Hanley J, Mah D, Mageras G, Hunt M, Toner S, et al. The deep inspiration breath-hold technique in the treatment of inoperable non-small-cell lung cancer. Int J Radiat Oncol Biol Phys. 2000;48:81-7.

25. Osman SOS, Hol S, Poortmans PM, Essers M. Volumetric modulated arc therapy and breath-hold in image-guided locoregional left-sided breast irradiation. Radiother Oncol. 2014;112:17-22.

26. Grantzau T, Overgaard J. Risk of second non-breast cancer after radiotherapy for breast cancer: a systematic review and meta-analysis of 762,468 patients. Radiother Oncol. 2015;114:56-65.

27. Hall EJ, Wuu C-S. Radiation-induced second cancers: the impact of 3D-CRT and IMRT. Int J Radiat Oncol Biol Phys. 2003;56:83-8.

28. Pan CC, Kavanagh BD, Dawson LA, Li XA, Das SK, Miften M, et al. Radiationassociated liver injury. Int J Radiat Oncol Biol Phys. 2010;76(Suppl 3):94-100. 
29. Prabhakar R, Tharmar G, Julka PK, Rath GK, Joshi RC, Bansal AK, et al. Impact of different breathing conditions on the dose to surrounding normal structures in tangential field breast radiotherapy. J Med Phys. 2007;32:24-8.

30. Altınok A, Askeroğlu O, Doyuran M, Çağlar M, Cantürk E, Erol C, et al. Dosimetric evaluation of right coronary artery in radiotherapy for breast cancer. Med Dosim. 2019;44:205-9.

31. Tang X, Zagar TM, Bair E, Jones EL, Fried D, Zhang L, et al. Clinical experience with 3-dimensional surface matching-based deep inspiration breath hold for left-sided breast cancer radiation therapy. Pract Radiat Oncol. 2014;4:151-8.

32. Jensen C, Urribarri J, Cail D, Rottmann J, Mishra P, Lingos T, et al. Cine EPID evaluation of two non-commercial techniques for DIBH. Med Phys. 2014;41:021730.

33. Macrie BD, Donnelly ED, Hayes JP, Gopalakrishnan M, Philip RT, Reczek J, et al. A cost-effective technique for cardiac sparing with deep inspirationbreath hold (DIBH). Phys Med. 2015;31:733-7.

34. Rong Y, Walston S, Welliver MX, Chakravarti A, Quick AM. Improving intrafractional target position accuracy using a 3D surface surrogate for left breast irradiation using the respiratory-gated deep-inspiration breath-hold technique. PLoS One. 2014;9:97933.

35. Jensen CA, Abramova T, Frengen J, Lund J-Å. Monitoring deep inspiration breath hold for left-sided localized breast cancer radiotherapy with an inhouse developed laser distance meter system. J Appl Clin Med Phys. 2017; 18(5):117-23.

36. Tanna N, McLauchlan R, Karis S, Welgemoed C, Gujral DM, Cleator SJ. Assessment of upfront selection criteria to prioritise patients for breath-hold left-sided breast radiotherapy. Clin Oncol. 2017;29:356-61.

37. Dell'Oro M, Giles E, Sharkey A, Borg M, Connell C, Bezak E. A retrospective dosimetric study of radiotherapy patients with left-sided breast cancer; patient selection criteria for deep inspiration breath hold technique. Cancers (Basel). 2019;11:259.

38. Ho AY, Ballangrud A, Li G, Gupta GP, McCormick B, Gewanter R, et al. Longterm pulmonary outcomes of a feasibility study of inverse-planned, multibeam intensity modulated radiation therapy in node-positive breast cancer patients receiving regional nodal irradiation. Int J Radiat Oncol Biol Phys. 2019;103:1100-8.

39. Lind PA, Wennberg B, Gagliardi G, Rosfors S, Blom-Goldman U, Lideståhl A et al. ROC curves and evaluation of radiation-induced pulmonary toxicity in breast cancer. Int J Radiat Oncol Biol Phys. 2006;64:765-70.

40. Wang J, Hu W, Yang Z, Chen X, Wu Z, Yu X, et al. Is it possible for knowledge-based planning to improve intensity modulated radiation therapy plan quality for planners with different planning experiences in leftsided breast cancer patients? Radiat Oncol. 2018;12:85.

\section{Publisher's Note}

Springer Nature remains neutral with regard to jurisdictional claims in published maps and institutional affiliations.

Ready to submit your research? Choose BMC and benefit from:

- fast, convenient online submission

- thorough peer review by experienced researchers in your field

- rapid publication on acceptance

- support for research data, including large and complex data types

- gold Open Access which fosters wider collaboration and increased citations

- maximum visibility for your research: over $100 \mathrm{M}$ website views per year

At $\mathrm{BMC}$, research is always in progress.

Learn more biomedcentral.com/submissions 